\title{
Increased Difficulties to Control Late Blight in Tunisia Are Caused by a Genetically Diverse Phytophthora infestans Population Next to the Clonal Lineage NA-01
}

Kalthoum Harbaoui, Genetic and Plant Breeding, Institute National Agronomic of Tunisia, Tunis, Tunisia and Regional Field Crops Research Centre Béja, Tunisia; Walid Hamada, Genetic and Plant Breeding, Institute National Agronomic of Tunisia; Ying Li, Institute of Vegetables and Flowers (IVF), CAAS, Beijing; Vivianne G. A. A. Vleeshouwers, Wageningen UR Plant Breeding, Wageningen, The Netherlands; and Theo van der Lee, Plant Research International B.V., Wageningen UR Biointeraction and Plant Health, The Netherlands

\begin{abstract}
Harbaoui, K., Hamada, W., Li, Y., Vleeshouwers, V. G. A. A., and van der Lee, T. 2014. Increased difficulties to control late blight in Tunisia are caused by a genetically diverse Phytophthora infestans population next to the clonal lineage NA-01. Plant Dis. 98:898-908.

In Tunisia, late blight caused by Phytophthora infestans is a serious threat to potato and tomato. The Mediterranean weather conditions can be conducive to infection in all seasons and the host crops, tomato and potato, are grown year round. Potato is planted and harvested in two to four overlapping intervals from August to June and tomato is grown both in open fields and in greenhouses. The consequences of these agricultural practices and the massive import of seed potato on the genetic variation of $P$. infestans are largely unknown. We conducted a survey in which $165 P$. infestans isolates, collected from five subregions in Tunisia between 2006 and 2008, on which we studied genotypic diversity through nuclear (simple-sequence repeat [SSR]) markers and combined this with a previous study on their mitochondrial haplotypes (mtDNA). The phylogenetic analysis revealed the presence of a major clonal lineage (NA-01, A1 mating type, mitochondrial haplotype Ia). Isolates belonging to this clonal lineage were found in

all regions and showed a relatively simple virulence pattern on a potato differential set carrying different Solanum demissum resistance genes. Apart from isolates belonging to this NA-01 clonal lineage, a group of isolates was found that showed a high genetic diversity, comprising both mating types and a more complex race structure that was found in the regions where late blight on potato was more difficult to control. The population on potato and tomato seems to be under different selection pressures. Isolates collected from tomato showed a low genetic diversity even though potato isolates collected simultaneously from the same location showed a high genetic diversity. Based on the SSR profile comparison, we could demonstrate that the four major clonal lineages found in the Netherlands and also in other European countries could not be found in Tunisia. Despite the massive import of potato seed from Europe, the P. infestans population in Tunisia was found to be clearly distinct.
\end{abstract}

Phytophthora infestans (Mont.) de Bary is the causal agent of late blight on potato and tomato $(9,14)$ and resulted in the dramatic epidemic leading to the potato Irish famine in 1845 (35). P. infestans, belonging to the oomycetes, is diploid during its vegetative life cycle and has two mating types (A1 and A2). Late blight is a major constraint for potato and tomato production (17) and epidemics have to be controlled by chemicals, resistant cultivars, and agricultural practices. Efficacy of most resistance genes introgressed from wild Solanum spp. to cultivated potato was quickly lost due to adaptations in $P$. infestans populations (25). Therefore, potato-producing countries in the world still suffer from late blight damages.

In Tunisia, late blight has become more difficult to control during the last decade and late blight epidemics can result in complete crop loss $(8,19)$. Agricultural practices in Tunisia result in the presence of potato plants in the field for up to 10 months of the year, comprising two to four partly overlapping seasons. The main potato cropping season spans from March to July, the late season from September to December, the very early season from November to February, and the early season from December to March. In Tunisia, growing potato in the main and late seasons is common practice in all major production regions but the early and the very

Corresponding author: T. A. J. van der Lee, E-mail: theo.vanderlee@wur.nl

* The $e$-Xtra logo stands for "electronic extra" and indicates that a supplementary table is available online.

Accepted for publication 12 December 2013.

http://dx.doi.org/10.1094/PDIS-06-13-0610-RE

(C) 2014 The American Phytopathological Society early season are specific for the Northeast and the Coastal regions. In Tunisia, $P$. infestans also causes severe late blight epidemics on tomato, either on greenhouses crops or field crops in the most productive regions. Commercial production of tomato and potato can be found in adjacent plots in many regions. Sometimes, potato and tomato are grown on the same farm or even in the same field. Drip and sprinkler irrigation, often used in tomato and potato farming in Tunisia, provide favorable conditions even in dry seasons. Nevertheless, rainy years correlate strongly with a high index of late blight and a reduction of the annual crop production. During the late-season crop, the climate is most conducive to the development of late blight epidemics due to the occurrence of clouded skies and rainy weather. As in most other regions in the world, in Tunisia, late blight is controlled by the use of chemicals $(9,26)$. However, this strategy may not be durable because resistant strains have been identified in many regions worldwide $(5,26,30)$.

Although clonal populations of $P$. infestans are able to cause severe diseases on crops, aggravation of the problems can be associated with the arrival of genetically diverse populations $(2,18)$. However, in Tunisia, little is known about the genetic diversity of $P$. infestans and other epidemiologic aspects. The first report of the presence of the A2 mating type in Tunisia (19) indicates that the population may be changing. More support for population changes came from the screening of resistance levels on 16 potato varieties against $P$. infestans by Ferjaoui et al. (8), in which more complex virulence patterns were found.

Recently, we reported on the characterization of $P$. infestans isolates derived from a national survey based on phenotypic characterization and mitochondrial genotypes (15). Therefore, populations of $P$. infestans never revealed phylogenetic lineages evading Tunisian crops. This prompted a comprehensive study on the genetic diversity using nuclear markers. We applied the recently described Simple-Sequence Repeat (SSR) markers (21) to genetically 
characterize the Tunisian population and demonstrate (i) the genetic diversity, population structure of the Tunisian $P$. infestans population, and phylogenetic lineages; (ii) the correlation between genetic diversity, virulence structure, and fungicide resistance in Tunisian population; (iii) the selection pressure occurring in $P$. infestans populations depending on host; and (iv) the specificity of the Tunisian population compared with Europe and North African areas.

\section{Materials and Methods}

Sampling. Samples were collected from potato crops and tomato greenhouses and fields from five major crop regions in Tunisia (Fig. 1): North (Bizerte), Northeast (Nabeul), Northwest (Beja and Jendouba), Tunis area, and Coastal Zone (Chott Meriem). We focused on a wide sampling from major potato crop regions in the North of Tunisia collected during 2005, 2006, 2007, and 2008 (Table 1). Mating type identification was reported previously (15). Four isolates (TU0501, TU0502, TU0503, and TU0504) collected at the end of December 2005 were analyzed with the 2006 population. Samples were collected from two hosts (potato and tomato) at different times in one season, in different seasons, from different fields, and also from the same field (Table 1). In the same field, two sampling strategies were used. The first one was to collect a sample from each of the four corners in a field with the same culture condition (cultivar, fungicide treatment, and irrigation). The second strategy was to collect from potato varieties cultivated in the same field that differ in the level of resistance to P. infestans.

Isolation and storage. Small pieces from the biotrophic part of blighted samples (leaf, stem, tuber, and fruit) freshly collected from fields or greenhouses were placed in petri dishes under disinfected tuber slices and incubated at $18^{\circ} \mathrm{C}$ and $16 \mathrm{~h}$ in light and $8 \mathrm{~h}$ in darkness for 6 to 7 days in a climate chamber. When mycelium was growing on the top of the potato slice, the mycelium was transferred to fresh rye agar medium amended with rifampicin (24 $\mathrm{mg}$ liter $\left.^{-1}\right)$. $P$. infestans mycelia were purified by repetitive transfers to rye agar medium amended with rifampicin after microscopic checks. Pure cultures were maintained at $18^{\circ} \mathrm{C}$ in the dark until further use. For long-term storage, two plugs of pure and fresh mycelia were placed in a cryotube vial $(1.8 \mathrm{ml})$ filled with $15 \%$ dimethyl sulfoxide solution. Vials were frozen slowly (in a Polystyrene box) at $-80^{\circ} \mathrm{C}$ during $24 \mathrm{~h}$ and transferred to the liquid nitrogen container. To retransfer strains into fresh medium, vials can be taken from liquid nitrogen and kept at room temperature until thawing, and plugs can be placed on medium plates.

DNA extraction. To produce mycelia, $1-\mathrm{cm}^{2}$ plugs of pure mycelium were placed in a petri dish filled with $20 \mathrm{ml}$ of liquid pea broth. After 5 to 7 days of incubation, mycelia was rinsed with sterile distilled water, filtered to remove excess of liquid, and dried in a flow cabinet for $30 \mathrm{~min}$. Approximately 50 to $100 \mathrm{mg}$ of mycelium was used for DNA extraction using a protocol from the International Potato Centre (www.cipotato.org). Genomic DNA extracts were stored at $-20^{\circ} \mathrm{C}$ until use.

SSR markers. Of the 165 collected and purified isolates, 157 were analyzed with 12 SSR markers using the multiplex SSR set described by Li et al. $(21,22)$. The resulting amplification products were sized by capillary electrophoresis on an automated ABI 3730 using the molecular standard GeneScan-500 ROX and scored using GeneMapper3.7 software (Applied Biosystems).

Data analysis. Genetic diversity based on SSR data was estimated according to the number of total alleles. The gene diversity and the genetic distances between populations were computed according to Nei (27) using the POPGENE software, v.1.31 (36). The multilocus microsatellite genotype (MLMG) for each isolate and the number of MLMGs in each population were determined using the program MULTILOCUS V.1.3 (1). Isolates with the same MLMG were treated as clones. Several indices of clonal diversity

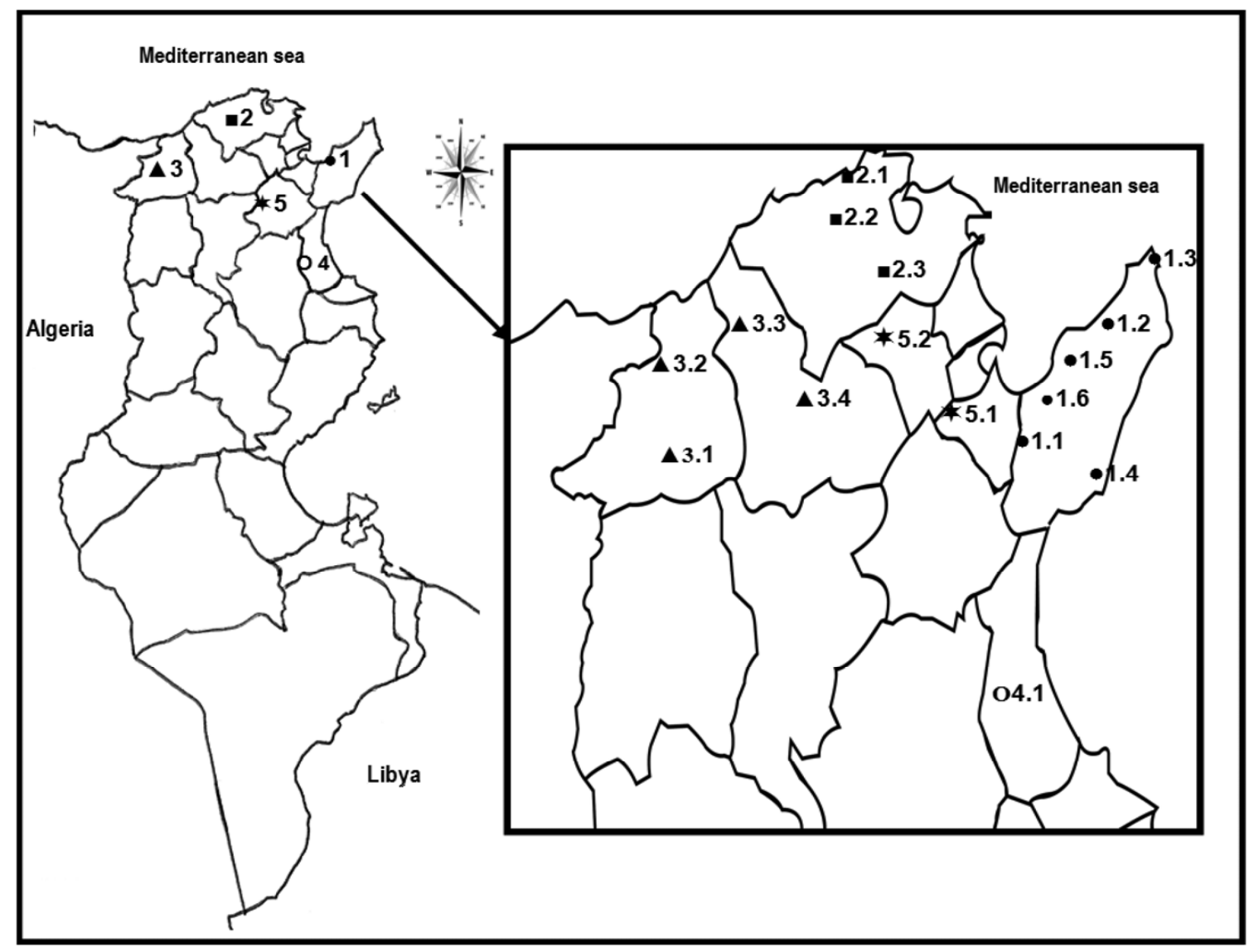

Fig. 1. Tunisian map showing different sampling subregions. $\bullet=$ Northeast (1): $1.1=$ Northeast $\mathrm{S}, 1.2=$ Northeast $\mathrm{Da}, 1.3=$ Northeast $\mathrm{H}, 1.4=$ Northeast $\mathrm{K}, 1.5=$ Northeast T; $=$ North (2): $2.1=$ North A, $2.2=$ North RJ, $2.3=$ North GM; $\boldsymbol{\Delta}=$ Northwest (3): $3.1=$ Northwest BS, $3.2=$ Northwest J, $3.3=$ Northwest B, $3.4=$ Northwest G; $\bigcirc=$ Costal Zone (4): $4.1=$ Coastal CM; and $*=$ Tunis (5): $5.1=$ Tunis area M, $5.2=$ Tunis area Kh. 
Table 1. Tunisian isolates used in this study ${ }^{\mathrm{a}}$

\begin{tabular}{|c|c|c|c|c|c|c|c|c|c|}
\hline Isolate $^{b}$ & $\begin{array}{l}\text { Sampling } \\
\text { year }\end{array}$ & Location & $\begin{array}{c}\text { Crop } \\
\text { season }\end{array}$ & $\begin{array}{c}\text { Plant } \\
\text { host }\end{array}$ & $\begin{array}{c}\text { Organ } \\
\text { host }\end{array}$ & Virulence & Metalaxyl & $\begin{array}{c}\text { Mating } \\
\text { type }\end{array}$ & $\begin{array}{c}\text { mDNA } \\
\text { haplotype }\end{array}$ \\
\hline TU0775 & 2007 & Northeast B & Late & Potato & Leaf & - & $\mathrm{R}$ & $\mathrm{A} 1$ & Ia \\
\hline TU0612 & 2006 & Northeast DA & Season & Potato & Leaf & - & - & $\mathrm{A} 1$ & Ia \\
\hline TU0625 & 2006 & Northeast DA & Season & Potato & Leaf & 1.4 .6 & $\mathrm{R}$ & $\mathrm{A} 1$ & Ia \\
\hline TU0626 & 2006 & Northeast DA & Season & Potato & Leaf & - & $\mathrm{S}$ & A1 & Ia \\
\hline TU0501 & 2005 & Northeast H & Late & Potato & Leaf & - & - & $\mathrm{A} 1$ & Ia \\
\hline TU0636 & 2006 & Northeast $\mathrm{H}$ & Late & Potato & Leaf & - & - & A1 & Ia \\
\hline TU0637 & 2006 & Northeast $\mathrm{H}$ & Late & Potato & Leaf & - & - & $\mathrm{A} 1$ & Ia \\
\hline TU0638 & 2006 & Northeast $\mathrm{H}$ & Late & Potato & Leaf & - & - & $\mathrm{A} 1$ & Ia \\
\hline TU0639 & 2006 & Northeast $\mathrm{H}$ & Late & Potato & Leaf & - & - & A1 & Ia \\
\hline TU0640 & 2006 & Northeast $\mathrm{H}$ & Late & Potato & Leaf & - & $\mathrm{S}$ & $\mathrm{A} 1$ & Ia \\
\hline TU0641 & 2006 & Northeast $\mathrm{H}$ & Late & Potato & Leaf & - & - & A1 & Ia \\
\hline TU0642 & 2006 & Northeast $\mathrm{H}$ & Late & Potato & Leaf & - & $\mathrm{R}$ & $\mathrm{A} 1$ & Ia \\
\hline TU0643 & 2006 & Northeast H & Late & Potato & Leaf & - & - & $\mathrm{A} 1$ & Ia \\
\hline TU0644 & 2006 & Northeast $\mathrm{H}$ & Late & Potato & Leaf & - & - & $\mathrm{A} 1$ & Ia \\
\hline TU0605 & 2006 & Northeast K & Early & Potato & Leaf & - & - & $\mathrm{A} 1$ & Ia \\
\hline TU0606 & 2006 & Northeast K & Early & Potato & Leaf & - & - & A1 & Ia \\
\hline TU0607 & 2006 & Northeast K & Early & Potato & Leaf & - & - & $\mathrm{A} 1$ & Ia \\
\hline TU0608 & 2006 & Northeast K & Early & Potato & Leaf & - & - & A1 & Ia \\
\hline TU0609 & 2006 & Northeast K & Early & Potato & Leaf & 1.2 .3 .4 .6 .10 .11 & $\mathrm{R}$ & A1 & Ia \\
\hline TU0610 & 2006 & Northeast K & Early & Potato & Leaf & - & $\mathrm{R}$ & A1 & Ia \\
\hline TU0615 & 2006 & Northeast K & Early & Potato & Leaf & - & - & $\mathrm{A} 1$ & Ia \\
\hline TU0616 & 2006 & Northeast K & Early & Potato & Leaf & - & - & $\mathrm{A} 1$ & Ia \\
\hline TU0617 & 2006 & Northeast K & Early & Potato & Leaf & - & $\mathrm{S}$ & $\mathrm{A} 1$ & Ia \\
\hline TU0619 & 2006 & Northeast K & Early & Potato & Leaf & 4.8 & $\mathrm{R}$ & $\mathrm{A} 2$ & Ia \\
\hline TU0620 & 2006 & Northeast K & Early & Potato & Leaf & - & - & A1 & Ia \\
\hline TU0623 & 2006 & Northeast K & Early & Potato & Leaf & - & $\mathrm{R}$ & $\mathrm{A} 2$ & Ia \\
\hline TU0624 & 2006 & Northeast K & Early & Potato & Leaf & - & - & A2 & Ia \\
\hline TU0627 & 2006 & Northeast K & Season & Potato & Leaf & - & - & $\mathrm{A} 2$ & Ia \\
\hline TU0756 & 2007 & Northeast K & Late & Potato & Leaf & 1.6 .7 & $\mathrm{R}$ & $\mathrm{A} 2$ & Ia \\
\hline TU0757 & 2007 & Northeast K & Late & Potato & Leaf & - & $\mathrm{R}$ & A1 & Ia \\
\hline TU0767 & 2007 & Northeast K & Early & Potato & Leaf & - & - & $\mathrm{A} 1$ & Ia \\
\hline TU0768 & 2007 & Northeast K & Early & Potato & Leaf & - & - & A1 & Ia \\
\hline TU0769 & 2007 & Northeast K & Early & Potato & Leaf & - & - & A1 & Ia \\
\hline TU0770 & 2007 & Northeast K & Early & Potato & Leaf & - & - & A1 & Ia \\
\hline TU0776 & 2007 & Northeast K & Late & Potato & Leaf & - & $\mathrm{R}$ & A2 & $\mathrm{Ia}$ \\
\hline TU0777 & 2007 & Northeast K & Late & Potato & Stem & - & - & $\mathrm{A} 1$ & Ia \\
\hline TU0778 & 2007 & Northeast K & Late & Potato & Leaf & - & - & A1 & Ia \\
\hline TU0779 & 2007 & Northeast K & Late & Potato & Leaf & 1.4 & $\mathrm{R}$ & $\mathrm{A} 2$ & Ia \\
\hline TU0780 & 2007 & Northeast K & Late & Potato & Leaf & - & - & A1 & Ia \\
\hline TU0781 & 2007 & Northeast K & Late & Potato & Stem & - & S & A1 & Ia \\
\hline TU0782 & 2007 & Northeast K & Late & Potato & Leaf & - & - & $\mathrm{A} 1$ & Ia \\
\hline TU0783 & 2007 & Northeast K & Late & Potato & Leaf & - & - & $\mathrm{A} 1$ & Ia \\
\hline TU0789 & 2007 & Northeast K & Late & Potato & Leaf & 1.3.4.6.10.11 & $\mathrm{R}$ & $\mathrm{A} 1$ & Ia \\
\hline TU0790 & 2007 & Northeast K & Late & Potato & Leaf & 1.3.4.7.10.11 & $\mathrm{R}$ & A1 & Ia \\
\hline TU0791 & 2007 & Northeast K & Late & Potato & Leaf & - & - & A1 & Ia \\
\hline TU0804 & 2008 & Northeast K & Early & Potato & Leaf & - & - & A2 & Ia \\
\hline TU0805 & 2008 & Northeast K & Early & Potato & Stem & - & - & $\mathrm{A} 1$ & Ia \\
\hline TU0806 & 2008 & Northeast K & Early & Potato & Stem & 1 & $\mathrm{R}$ & A2 & Ia \\
\hline TU0807 & 2008 & Northeast K & Early & Potato & Leaf & - & - & $\mathrm{A} 1$ & Ia \\
\hline TU0810 & 2008 & Northeast K & Season & Potato & Leaf & - & $\mathrm{R}$ & $\mathrm{A} 1$ & Ia \\
\hline TU0811 & 2008 & Northeast K & Season & Potato & Leaf & - & - & A1 & Ia \\
\hline TU0812 & 2008 & Northeast K & Season & Potato & Leaf & - & - & $\mathrm{A} 1$ & Ia \\
\hline TU0813 & 2008 & Northeast K & Season & Potato & Leaf & - & - & A1 & Ia \\
\hline TU0814 & 2008 & Northeast K & Season & Potato & Stem & - & - & A1 & Ia \\
\hline TU0815 & 2008 & Northeast K & Season & Potato & Leaf & - & - & A1 & Ia \\
\hline TU0816 & 2008 & Northeast K & Season & Potato & Leaf & 1.4 .6 & $\mathrm{R}$ & $\mathrm{A} 1$ & Ia \\
\hline TU0817 & 2008 & Northeast K & Season & Potato & Leaf & - & - & $\mathrm{A} 1$ & Ia \\
\hline TU0818 & 2008 & Northeast K & Season & Potato & Leaf & 3.7 & $\mathrm{~S}$ & A1 & Ia \\
\hline TU0819 & 2008 & Northeast K & Season & Potato & Leaf & - & - & A1 & Ia \\
\hline TU0820 & 2008 & Northeast K & Season & Potato & Leaf & - & $\mathrm{R}$ & A2 & Ia \\
\hline TU0821 & 2008 & Northeast K & Season & Potato & Leaf & - & - & $\mathrm{A} 2$ & Ia \\
\hline TU0822 & 2008 & Northeast K & Season & Potato & Leaf & - & $\mathrm{R}$ & A1 & Ia \\
\hline TU0823 & 2008 & Northeast K & Season & Potato & Leaf & - & - & A1 & $\mathrm{Ia}$ \\
\hline TU0824 & 2008 & Northeast K & Season & Potato & Leaf & - & - & $\mathrm{A} 1$ & Ia \\
\hline TU0825 & 2008 & Northeast K & Season & Potato & Leaf & - & $\mathrm{R}$ & A2 & Ia \\
\hline TU0826 & 2008 & Northeast K & Season & Potato & Leaf & - & - & A1 & Ia \\
\hline TU0827 & 2008 & Northeast K & Season & Potato & Leaf & 1.4 & $\mathrm{R}$ & A1 & Ia \\
\hline TU0828 & 2008 & Northeast K & Season & Potato & Leaf & - & - & $\mathrm{A} 2$ & $\mathrm{Ia}$ \\
\hline \multirow[t]{2}{*}{ TU0829 } & 2008 & Northeast K & Season & Potato & Leaf & - & - & $\mathrm{A} 2$ & Ia \\
\hline & & & & & & & & \multicolumn{2}{|c|}{ (continued on next page) } \\
\hline
\end{tabular}

${ }^{a} \mathrm{~S}=$ sensitive, $\mathrm{R}=$ resistant, and $-=$ not tested. Location names are described in detail in Figure 1.

${ }^{\mathrm{b}}$ Name meaning (TUXXYY): TU = Tunisia, XX = year of sampling, YY = number of the isolate. TU0814-TU0835, TU0848-TU0860, and TU0862TU0866 were sampled based on the second strategy of sampling and the remaining isolates were sampled based on the first strategy of sampling (both described in Materials and Methods). 
Table 1. (continued from preceding page)

\begin{tabular}{|c|c|c|c|c|c|c|c|c|c|}
\hline Isolate $^{b}$ & $\begin{array}{c}\text { Sampling } \\
\text { year }\end{array}$ & Location & $\begin{array}{c}\text { Crop } \\
\text { season }\end{array}$ & $\begin{array}{c}\text { Plant } \\
\text { host }\end{array}$ & $\begin{array}{c}\text { Organ } \\
\text { host }\end{array}$ & Virulence & Metalaxyl & $\begin{array}{l}\text { Mating } \\
\text { type }\end{array}$ & $\begin{array}{c}\text { mDNA } \\
\text { haplotype }\end{array}$ \\
\hline TU0830 & 2008 & Northeast K & Season & Potato & Leaf & - & - & A1 & Ia \\
\hline TU0831 & 2008 & Northeast K & Season & Potato & Leaf & - & - & A1 & $\mathrm{Ia}$ \\
\hline TU0832 & 2008 & Northeast K & Season & Potato & Leaf & - & - & A1 & $\mathrm{Ia}$ \\
\hline TU0833 & 2008 & Northeast K & Season & Potato & Leaf & - & - & A1 & $\mathrm{Ia}$ \\
\hline TU0834 & 2008 & Northeast K & Season & Potato & Leaf & - & - & A1 & Ia \\
\hline TU0835 & 2008 & Northeast K & Season & Potato & Leaf & - & - & A1 & Ia \\
\hline TU0836 & 2008 & Northeast K & Field crop & Tomato & Leaf & - & $\mathrm{S}$ & A1 & Ia \\
\hline TU0839 & 2008 & Northeast K & Field crop & Tomato & Stem & - & - & A1 & Ia \\
\hline TU0840 & 2008 & Northeast K & Field crop & Tomato & Leaf & - & $\mathrm{S}$ & A1 & Ia \\
\hline TU0862 & 2008 & Northeast K & Early & Potato & Leaf & 1 & $\mathrm{~S}$ & A1 & Ia \\
\hline TU0863 & 2008 & Northeast K & Early & Potato & Leaf & - & - & $\mathrm{A} 2$ & Ia \\
\hline TU0864 & 2008 & Northeast K & Early & Potato & Leaf & - & - & A1 & Ia \\
\hline TU0865 & 2008 & Northeast K & Early & Potato & Leaf & - & - & $\mathrm{A} 2$ & $\mathrm{Ia}$ \\
\hline TU0866 & 2008 & Northeast K & Early & Potato & Leaf & - & - & A1 & Ia \\
\hline TU0648 & 2006 & Northeast S & Late & Potato & Leaf & - & - & A1 & Ia \\
\hline TU0750 & 2007 & Northeast S & Late & Potato & Tuber & - & - & A1 & Ia \\
\hline TU0758 & 2007 & Northeast S & Early & Tomato & Leaf & 1.4.6.7 & $\mathrm{R}$ & A1 & Ia \\
\hline TU0808 & 2008 & Northeast S & Early & Tomato & Leaf & - & - & A1 & Ia \\
\hline TU0502 & 2005 & Northeast T & Late & Potato & Leaf & - & - & A1 & Ia \\
\hline TU0613 & 2006 & Northeast T & Early & Tomato & Leaf & - & $\mathrm{S}$ & A1 & IIa \\
\hline TU0614 & 2006 & Northeast T & Early & Tomato & Leaf & 7 & $\mathrm{R}$ & A1 & IIa \\
\hline TU0618 & 2006 & Northeast T & Early & Potato & Leaf & 1.3.6.7 & $\mathrm{S}$ & A1 & Ia \\
\hline TU0621 & 2006 & Northeast T & Early & Tomato & Leaf & - & $\mathrm{S}$ & A1 & IIa \\
\hline TU0622 & 2006 & Northeast T & Early & Tomato & Leaf & - & - & A1 & IIa \\
\hline TU0646 & 2006 & Northeast T & Early & Tomato & Leaf & 1.4 .6 & $\mathrm{R}$ & A1 & Ia \\
\hline TU0647 & 2006 & Northeast T & Early & Tomato & Leaf & - & - & A1 & Ia \\
\hline TU0649 & 2006 & Northeast T & Early & Tomato & Leaf & - & $\mathrm{S}$ & A1 & $\mathrm{Ia}$ \\
\hline TU0751 & 2007 & Northeast T & Early & Tomato & Leaf & - & - & A1 & Ia \\
\hline TU0752 & 2007 & Northeast T & Early & Tomato & Stem & - & - & A1 & Ia \\
\hline TU0753 & 2007 & Northeast T & Early & Tomato & leaf & - & - & A1 & Ia \\
\hline TU0754 & 2007 & Northeast T & Early & Tomato & Stem & - & - & A1 & Ia \\
\hline TU0755 & 2007 & Northeast T & Early & Tomato & Leaf & - & - & A1 & Ia \\
\hline TU0759 & 2007 & Northeast T & Early & Tomato & Leaf & - & $\mathrm{R}$ & A1 & Ia \\
\hline TU0760 & 2007 & Northeast T & Early & Tomato & Leaf & - & - & A1 & Ia \\
\hline TU0761 & 2007 & Northeast T & Early & Tomato & Stem & - & - & A1 & Ia \\
\hline TU0762 & 2007 & Northeast T & Early & Tomato & Leaf & - & $\mathrm{S}$ & A1 & Ia \\
\hline TU0763 & 2007 & Northeast T & Early & Tomato & Leaf & - & - & A1 & Ia \\
\hline TU0764 & 2007 & Northeast T & Early & Tomato & Stem & - & - & A1 & Ia \\
\hline TU0765 & 2007 & Northeast T & Early & Tomato & Leaf & 1.7 & $\mathrm{~S}$ & A1 & $\mathrm{Ia}$ \\
\hline TU0766 & 2007 & Northeast T & Early & Tomato & Leaf & - & - & A1 & Ia \\
\hline TU0771 & 2007 & Northeast T & Early & Tomato & Leaf & - & - & A1 & Ia \\
\hline TU0773 & 2007 & Northeast T & Early & Tomato & Leaf & - & - & A1 & Ia \\
\hline TU0774 & 2007 & Northeast T & Early & Tomato & Leaf & - & $\mathrm{S}$ & A1 & Ia \\
\hline TU0611 & 2006 & North A & Season & Potato & Leaf & - & $\mathrm{R}$ & A1 & Ia \\
\hline TU0628 & 2006 & North GM & Season & Potato & Leaf & - & $\mathrm{S}$ & A1 & Ia \\
\hline TU0629 & 2006 & North GM & Season & Potato & Leaf & 1.3.6.7.11 & $\mathrm{R}$ & A1 & Ia \\
\hline TU0630 & 2006 & North GM & Season & Potato & Leaf & 1 & $\mathrm{R}$ & A1 & Ia \\
\hline TU0631 & 2006 & North GM & Season & Potato & Leaf & - & - & A1 & Ia \\
\hline TU0632 & 2006 & North GM & Season & Potato & Leaf & - & - & A1 & Ia \\
\hline TU0633 & 2006 & North GM & Season & Potato & Leaf & - & - & A1 & Ia \\
\hline TU0634 & 2006 & North GM & Season & Potato & Leaf & - & $\mathrm{R}$ & A2 & $\mathrm{Ia}$ \\
\hline TU0635 & 2006 & North GM & Season & Potato & Leaf & - & $\mathrm{S}$ & A1 & Ia \\
\hline TU0792 & 2007 & North GM & Late & Potato & Leaf & - & - & A1 & $\mathrm{Ia}$ \\
\hline TU0793 & 2007 & North GM & Late & Potato & Leaf & 1.2.4.6.7 & $\mathrm{R}$ & A2 & Ia \\
\hline TU0794 & 2007 & North GM & Late & Potato & Leaf & - & - & $\mathrm{A} 2$ & Ia \\
\hline TU0795 & 2007 & North GM & Late & Potato & Leaf & - & $\mathrm{R}$ & A2 & $\mathrm{Ia}$ \\
\hline TU0796 & 2007 & North GM & Late & Potato & Tuber & - & $\mathrm{S}$ & $\mathrm{A} 1$ & Ia \\
\hline TU0848 & 2008 & North GM & Late & Potato & Leaf & - & - & A2 & Ia \\
\hline TU0849 & 2008 & North GM & Late & Potato & Leaf & 0 & $\mathrm{~S}$ & $\mathrm{~A} 1$ & Ia \\
\hline TU0850 & 2008 & North GM & Late & Potato & Leaf & 1.2.3.7 & $\mathrm{R}$ & A1 & Ia \\
\hline TU0851 & 2008 & North GM & Late & Potato & Leaf & - & - & A1 & Ia \\
\hline TU0852 & 2008 & North GM & Late & Potato & Leaf & - & - & $\mathrm{A} 1$ & Ia \\
\hline TU0853 & 2008 & North GM & Late & Potato & Leaf & - & - & A1 & $\mathrm{Ia}$ \\
\hline TU0854 & 2008 & North GM & Late & Potato & Leaf & - & - & A1 & Ia \\
\hline TU0855 & 2008 & North GM & Late & Potato & Leaf & - & - & $\mathrm{A} 1$ & Ia \\
\hline TU0856 & 2008 & North GM & Late & Potato & Leaf & 1.2 .3 .7 .10 .11 & $\mathrm{R}$ & A2 & Ia \\
\hline TU0857 & 2008 & North GM & Late & Potato & Leaf & - & - & A1 & Ia \\
\hline TU0858 & 2008 & North GM & Late & Potato & Leaf & 1.3 .7 & $\mathrm{R}$ & A2 & Ia \\
\hline TU0859 & 2008 & North GM & Late & Potato & Stem & - & - & A1 & Ia \\
\hline TU0860 & 2008 & North GM & Late & Potato & Leaf & - & - & A2 & Ia \\
\hline TU0861 & 2008 & North GM & Late & Potato & Leaf & - & - & $\mathrm{A} 1$ & $\mathrm{Ia}$ \\
\hline TU0837 & 2008 & North RJ & Season & Potato & Leaf & - & - & A1 & Ia \\
\hline
\end{tabular}


Table 1. (continued from preceding page)

\begin{tabular}{|c|c|c|c|c|c|c|c|c|c|}
\hline Isolate $^{b}$ & $\begin{array}{c}\text { Sampling } \\
\text { year }\end{array}$ & Location & $\begin{array}{c}\text { Crop } \\
\text { season }\end{array}$ & $\begin{array}{c}\text { Plant } \\
\text { host }\end{array}$ & $\begin{array}{c}\text { Organ } \\
\text { host }\end{array}$ & Virulence & Metalaxyl & $\begin{array}{l}\text { Mating } \\
\text { type }\end{array}$ & $\begin{array}{c}\text { mDNA } \\
\text { haplotype }\end{array}$ \\
\hline TU0841 & 2008 & Northwest BS & Late & Potato & Leaf & - & - & A1 & Ia \\
\hline TU0842 & 2008 & Northwest BS & Late & Potato & Leaf & - & - & A1 & $\mathrm{Ia}$ \\
\hline TU0843 & 2008 & Northwest BS & Late & Potato & Leaf & 1.2.3.7 & $\mathrm{R}$ & A1 & Ia \\
\hline TU0844 & 2008 & Northwest BS & Late & Potato & Leaf & - & - & A1 & $\mathrm{Ia}$ \\
\hline TU0845 & 2008 & Northwest BS & Late & Potato & Leaf & - & - & A1 & Ia \\
\hline TU0846 & 2008 & Northwest BS & Late & Potato & Leaf & 0 & $\mathrm{R}$ & A1 & $\mathrm{Ia}$ \\
\hline TU0847 & 2008 & Northwest BS & Late & Potato & Leaf & - & $\mathrm{S}$ & A1 & Ia \\
\hline TU0785 & 2007 & Northwest G & Late & Potato & Leaf & 1.2.3.7 & $\mathrm{S}$ & A1 & Ia \\
\hline TU0786 & 2007 & Northwest G & Late & Potato & Leaf & 1.7 & $\mathrm{~S}$ & A1 & $\mathrm{Ia}$ \\
\hline TU0787 & 2007 & Northwest G & Late & Potato & Leaf & - & - & A1 & Ia \\
\hline TU0788 & 2007 & Northwest G & Late & Potato & Leaf & - & - & A1 & $\mathrm{Ia}$ \\
\hline TU0645 & 2006 & Northwest J & Late & Potato & Leaf & 1.2 & $\mathrm{~S}$ & A1 & Ia \\
\hline TU0897 & 2008 & Northwest J & Late & Potato & Stem & - & - & A1 & Ia \\
\hline TU0898 & 2008 & Northwest J & Late & Potato & Stem & - & - & A1 & $\mathrm{Ia}$ \\
\hline TU0899 & 2008 & Northwest J & Late & Potato & Leaf & 1.2 .7 & $\mathrm{R}$ & A1 & $\mathrm{Ia}$ \\
\hline TU0800 & 2008 & Northwest J & Late & Potato & Leaf & - & $\mathrm{S}$ & A1 & Ia \\
\hline TU0801 & 2008 & Northwest J & Late & Potato & Leaf & - & $\mathrm{R}$ & A1 & Ia \\
\hline TU0802 & 2008 & Northwest J & Late & Potato & leaf & - & - & A1 & Ia \\
\hline TU0803 & 2008 & Northwest J & Late & Potato & leaf & - & - & A1 & Ia \\
\hline TU0784 & 2007 & Northwest MB & Late & Potato & Stem & 0 & $\mathrm{~S}$ & A1 & Ia \\
\hline TU0604 & 2006 & Coastal CM & Late & Potato & Leaf & - & $\mathrm{S}$ & A1 & Ia \\
\hline TU0772 & 2007 & Coastal CM & Season & Potato & Leaf & - & $\mathrm{S}$ & A1 & Ia \\
\hline TU0503 & 2005 & Tunis M & Late & Potato & Leaf & - & - & A1 & Ia \\
\hline TU0809 & 2008 & Tunis Kh & Early & Tomato & Fruit & - & - & A1 & Ia \\
\hline
\end{tabular}

were calculated for each population, including (i) the number of different multilocus genotypes, (ii) the number of the most frequent genotype, and (iii) the clonal fraction (the proportion of isolates originating from asexual reproduction), calculated as 1 [(number of different genotypes)/(total number of isolates)] (37). MULTILOCUS V.1.3 was also used to compute the index of association $\left(\mathrm{I}_{\mathrm{A}}\right)$ and the correlation coefficient in order to deduce the possible linkage disequilibrium between alleles in the subpopulations and in the whole population. The occurrence of private alleles and the allele frequencies were computed using CONVERT software v. 1.31 (11). The genetic differentiation among P. infestans isolates collected from different regions and the gene flow were further investigated by the analysis of molecular variance using ARLEQUIN 3.0 software (7), applying a tolerance setting of 5\% missing values per locus. The SSR data were analyzed by the phylogenetic software package TREECON for Windows Version $1.3 \mathrm{~b}$ (32). Trees were rooted using an isolate with the US-1 genotype (VK1.4). Population structure was performed using a model-based software program, STRUCTURE 2.2 (29). In this model, a number of populations $(\mathrm{K}=1 \ldots 7)$ are assumed to be present, each of which is characterized by a set of allele frequencies at each locus. Individuals in the sample are assigned to populations (clusters), or jointly to more populations if their genotypes indicate that they are admixed. All loci are assumed to be independent, and each K population is assumed to follow Hardy-Weinberg equilibrium. The posterior probabilities were estimated using a Markov Chain Monte Carlo (MCMC) method. The MCMC chains were run at $10^{5}$ burn-in period lengths followed by $10^{6}$ iterations using a model allowing for admixture and correlated allele frequencies. However, the inference of the exact value of $\mathrm{K}$ (gene pool) was not straightforward, because the estimated log-likelihood values appeared to be an increasing function of $\mathrm{K}$ for all examined values of $\mathrm{K}$. Therefore, it may not be possible to find the true value of $\mathrm{K}$. In this situation, Campana and collaborators (3) propose to use CorrSieve software 1.6-3 that can calculate the $\Delta \mathrm{K}$ to determine a meaningful value of $K$. This program can summarize the $\Delta K$ statistic directly from STRUCTURE output files. The population $\mathrm{K}$ that has the highest value of $\Delta \mathrm{K}$ is considered as the meaningful population.

\section{Results}

Sample collection. In total, 165 isolates from over 500 tissue samples were successfully purified. Isolates comprise 112 isolates collected from potato and tomato in the Northeast region of Tunisia
(Nabeul), 29 isolates from the North of Tunisia (Bizerte) collected from potato fields, 20 isolates from the Northwest collected from potato fields, 2 isolates from the Coastal Zone collected from potato, 1 isolate from Tunis area (Manouba) collected from potato, and 1 isolate from Tunis area (Khoulaydia) collected from tomato.

Genotypic diversity. The isolates were grouped in four geographic regions: North, Northeast, Northeast T, and Northwest. We regarded the tomato isolates from the Northeast $\mathrm{T}$ as a separate group, because they represent the widest tomato sampling region in this study. Both the Tunis area and the Coastal Zone were eliminated from the genetic study on geographic distribution and STRUCTURE analysis because of the low number of individuals (two isolates) within each population but they were kept in the other analysis (host, mating type, and season analysis) and the phylogenetic clustering. After eliminating identical genotypes and samples that had missing data because of either polymerase chain reaction (PCR) failure or DNAs qualities, the total number of isolates was reduced from 157 to 130 individuals.

The genetic diversity based on geographic distribution of 126 of 130 individuals (4 individuals from the Coastal and Tunis areas are not included in this study) showed that the 12 SSR markers yielded 49 different alleles ranging from 2 (loci Pi07 and PinfSSR2) to 9 (G11) alleles per locus with a mean of 4 alleles per locus (Table 2). In all, 8 private alleles were detected within the 49 detected alleles, of which 6 were found in the Northeast, 1 in the North, and 1 in the Northwest. In the whole population, the gene diversity varied from 0.12 for marker D13 to 0.76 for marker G11, with an average value of 0.5 . The lowest value of gene diversity was detected in the North ( 0 for D13) and the highest value was detected in the Northeast ( 0.77 for G11). Within the 126 isolates, 72 distinct multilocus genotypes were detected, including 21 genotypes which are subclones (Table 3). A high genotypic diversity, ranging from 0.77 to 0.99 , was detected in all subpopulations. Overall, the clonal fraction was $43 \%$ but this varied strongly per region. The clonal fraction was low in nearly all regions (15 to 37\%) with the exception of the Northeast $\mathrm{T}(61 \%)$ that also showed the lowest genotypic diversity $(=0.77)$ and the highest value of $\mathrm{I}_{\mathrm{A}}$.

The phylogenetic tree constructed by the neighbor-joining method using Nei's genetic distances showed the presence of two phylogenetic clusters. Cluster I consists of a dominant clonal lineage designated as North Africa-01 (NA-01) and some related genotypes. The clonal lineage NA-01 groups 54 isolates, within which 21 subclonal genotypes were identified named NA-01_01 to 
NA-01_21. The dominant subclonal lineage is NA-01_16, represented by 21 isolates, and other subclonal genotypes show minor differences due to loss of particular alleles or by changes in the repeat number at some hypervariable loci. All isolates of the NA-
01 clonal lineage also shared the A1 mating type and the Ia haplotype. Isolates of genotype NA-01 were collected from almost all subregions (Fig. 1) and originate from tomato and potato.

Remarkably, none of the 66 isolates collected from potato in

Table 2. Diversity of simple-sequence repeat loci used in this study (Allele's number, gene diversity, and genetic diversity) ${ }^{\mathrm{a}}$

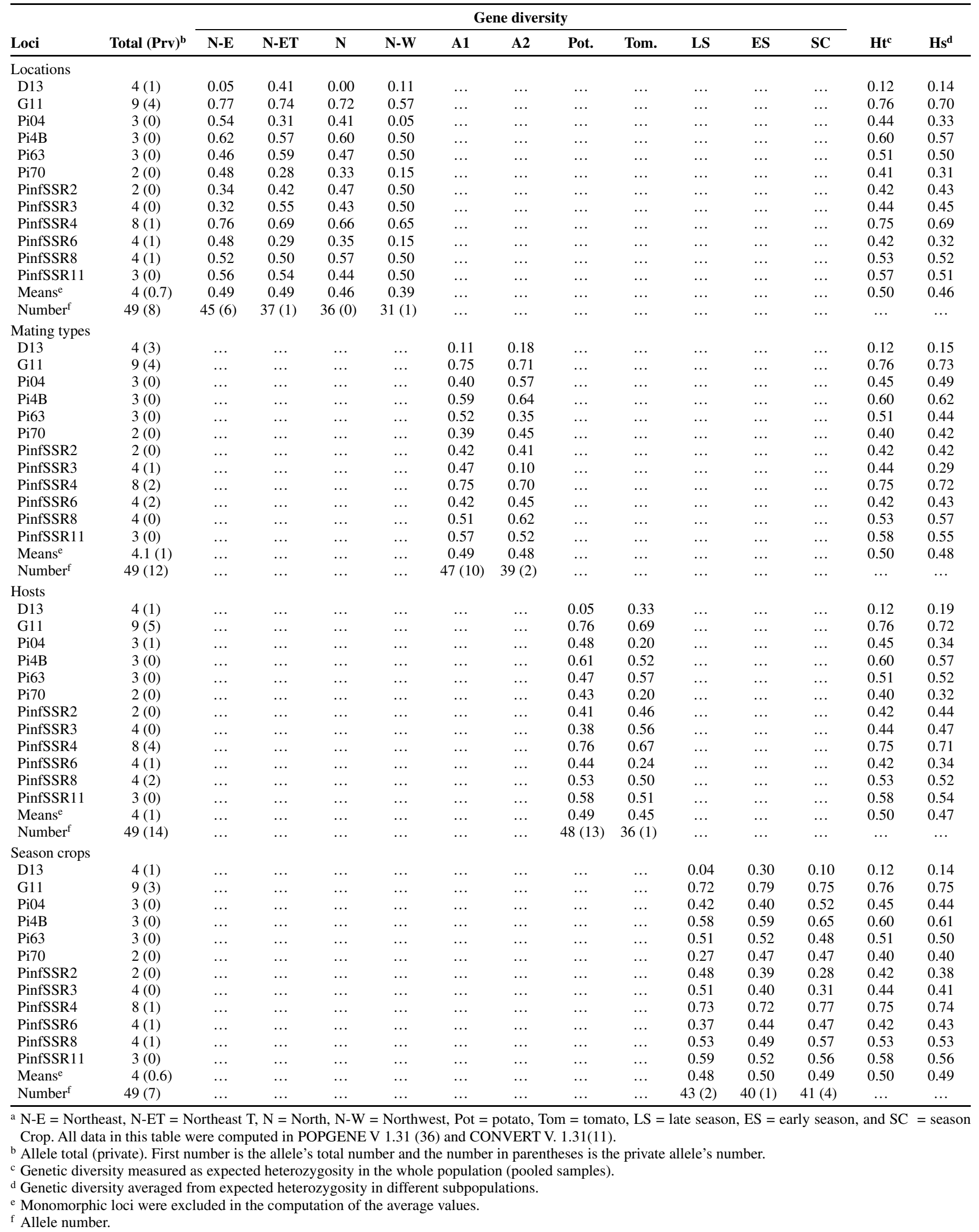


Northeast $\mathrm{K}$ belonged to this dominant clonal lineage. In contrast, the three tomato isolates from Northeast K (TU0836, TU0839, and TU0840) belong to NA-01. In addition to all members of the NA01 clonal lineage, three isolates (TU0645, TU0787, and TU0897) were grouped in cluster I (Fig. 2, marked by stars). In contrast to the members of the clonal lineage NA-01, these three isolates showed variations at almost all used SSR markers compared with NA-01. Also, they showed alternative alleles at two loci that are otherwise stable (PinfSSR11 and Pi70). Therefore, we concluded that these three isolates do not belong to the clonal lineage but may be related (discussed below). Cluster II consists of a genetically highly diverse group and comprises isolates with the A1 and the A2 mating type and two mtDNA haplotypes (Ia and IIa). Few clonal isolates were identified in cluster II. In this latter cluster, clonal isolates always originate from the same location, except for some isolates discussed in detail below. Almost all isolates in this cluster (68 of 73) were collected from potato. The exceptions are isolate TU0646, originating from tomato grown in a greenhouse (A1 mat-

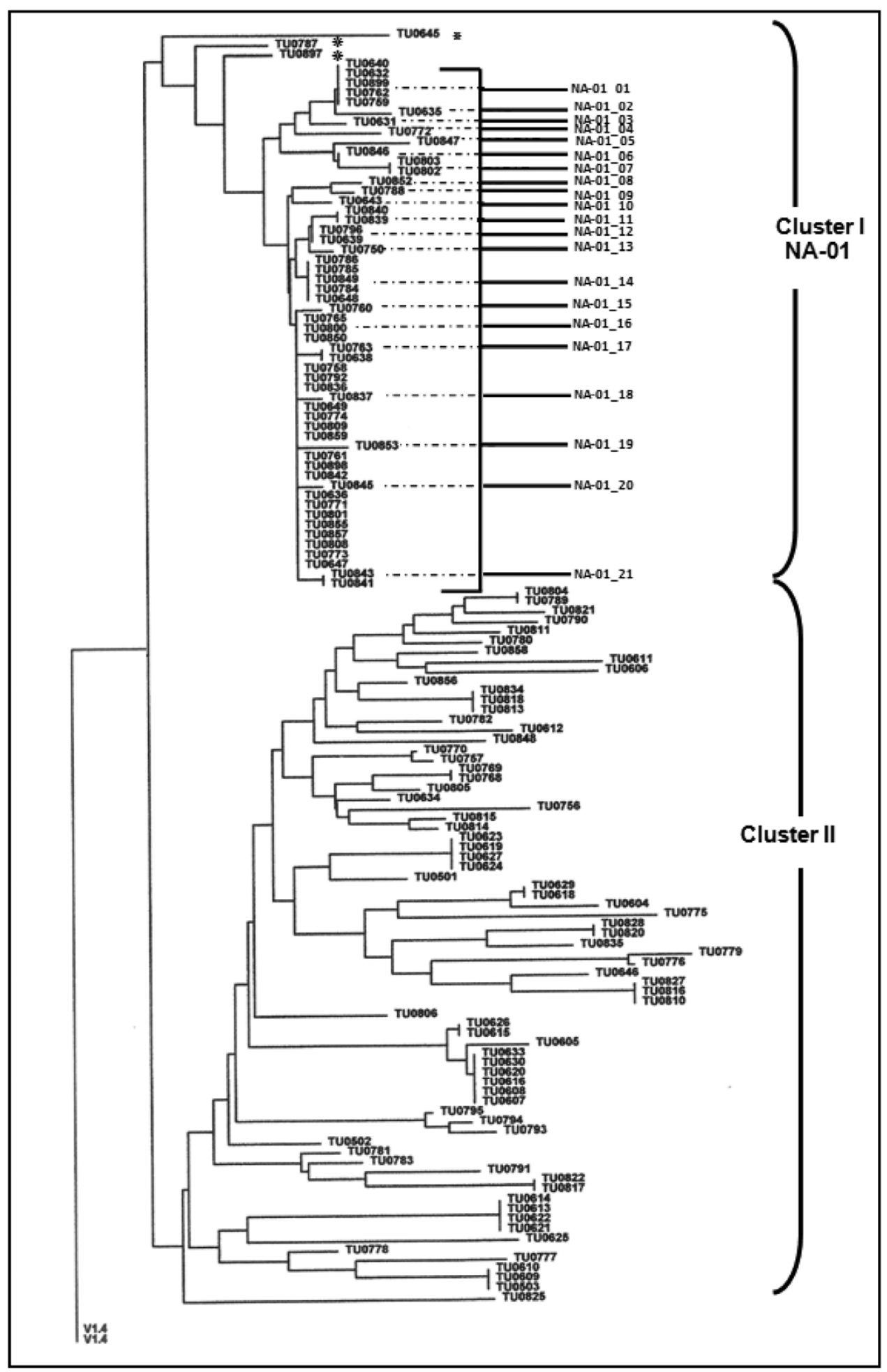

Fig. 2. Phylogenic tree of 130 individuals rooted by the universal isolate VK 1.4, computed by the neighbor-joining algorithm in TREECON for Windows Version 1.3b (32). Individual name (TUYYXX): TU = Tunisia, $Y Y=$ year of sampling, $X X=$ number of the isolate. Stars show three genotypes demonstrated by structure analysis as admixture forms. Vertical line in the bottom groups subclonal genotypes. Horizontal lines show different subclonal genotypes listed from NA-01_01 to NA-01_21. Vertical embraces in the right side show the two phylogenic clusters I and II. 
ing type and Ia haplotype), and a distinct group of four clonal isolates also collected from tomato grown in a greenhouse (TU0613, TU0614, TU0621, and TU0622), with the A1 mating type and an exceptional mitochondrial haplotype (IIa).

Population genetic structure. The analysis of the population genetic structure using 126 P. infestans isolates was carried out with the model-based STRUCTURE approach (29). $\mathrm{K}=2$ had the highest value of $\Delta \mathrm{K}$ (Fig. 3A) and two distinct gene pools were identified (Fig. 3B). The first inferred group (Fig. 3B, dark gray) represents the clonal lineage NA-01 detected in all sampling regions. The second inferred group (Fig. 3B, light gray) represents the genetic diverse group II. Of the 126 computed isolates, $123(98 \%)$ shared more than $90 \%$ membership with one of the two subpopulations and were classified as members of that population, whereas 3 isolates (TU0645, TU0787, and TU0897) were categorized as admixture forms with varying levels of

Table 3. Genotypic diversity and pairwise genetic differentiation (Fst) (above diagonal) and gene flow (Nm) (below diagonal) in Phytophthora infestans Tunisian subpopulations ${ }^{\mathrm{a}}$

\begin{tabular}{|c|c|c|c|c|c|c|c|c|c|c|}
\hline \multirow[b]{2}{*}{ Locations } & \multicolumn{6}{|c|}{ Genotypic diversity } & \multicolumn{4}{|c|}{ Genetic differentiation } \\
\hline & $\mathbf{N i}^{\mathbf{b}}$ & $\mathrm{Ng}^{\mathrm{c}}$ & $\mathrm{Nm}^{\mathrm{d}}$ & $\mathbf{G D}^{\mathrm{e}}$ & $\mathbf{I}_{\mathbf{A}}^{\mathbf{f}}$ & $\mathrm{CF}^{\mathrm{g}}$ & North & Northeast & Northeast T & Northwest \\
\hline North & 24 & 20 & 3 & 0.98 & 2.85 & 17 & 0.000 & $0.053 *$ & $0.028 *$ & $0.036^{*}$ \\
\hline Northeast & 65 & 45 & 4 & 0.99 & 1.06 & 31 & 8.947 & 0.000 & $0.143^{*}$ & $0.160^{*}$ \\
\hline Northeast T & 18 & 7 & 8 & 0.77 & 5.02 & 61 & 17.686 & 3.002 & 0.000 & $-0.015^{*}$ \\
\hline Northwest & 19 & 12 & 4 & 0.94 & 1.39 & 37 & 13.409 & 2.641 & -16.917 & 0.000 \\
\hline All samples & 126 & 72 & 20 & 0.97 & 2.43 & 43 & $\ldots$ & $\ldots$ & $\ldots$ & $\ldots$ \\
\hline
\end{tabular}

${ }^{\mathrm{a}} \mathrm{Fst}=0.05994^{*}, \mathrm{Nm}=0.25(1-\mathrm{Fst}) / \mathrm{Fst}=3.92$ (in all samples) computed using ARLEQUIN V.3.1 (7); $*$ indicates significant at $P<0.05$.

${ }^{\mathrm{b}}$ Number of individuals in each population.

${ }^{\mathrm{c}}$ Number of multilocus genotypes calculated by MULTILOCUS 1.3 (1).

${ }^{\mathrm{d}}$ Number of most frequent genotype calculated by MULTILOCUS 1.3 (1).

e Genotypic diversity computed by MULTILOCUS $1.3(1)$.

${ }^{\mathrm{f}}$ Index of association; in all data, correlation coefficient $\left(r_{\mathrm{d}}\right)$ is significant $\left(0.1<r_{\mathrm{d}}<0.4\right)$.

$\mathrm{g}$ Clonal fraction (\%) calculated according to Zhan et al. (37).

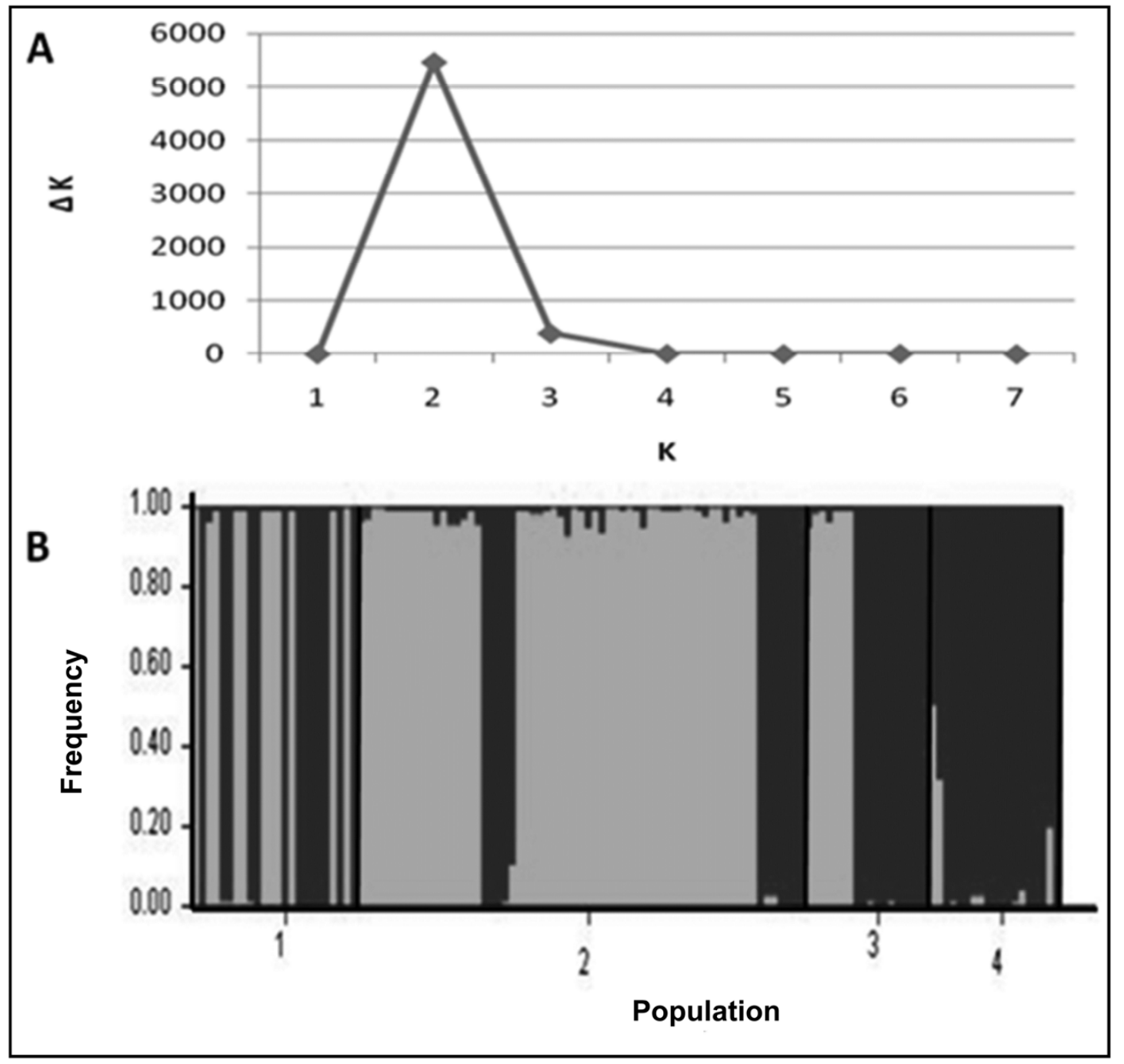

Fig. 3. A, $\Delta \mathrm{K}$ values of seven populations computed by CorrSieve program (3). B, Model-based ancestry of 126 Phytophthora infestans isolates using STRUCTURE 2.2 (29). Tones represent model-based subpopulations: light gray = clonal lineage NA-01 and dark gray = genetically diverse group (cluster II); lanes 1, 2, 3, and 4 represent sampling regions as following: $1=$ North, $2=$ Northeast, $3=$ Northeast $\mathrm{T}$, and $4=$ Northwest. 
membership shared among the two subpopulations. These potentially admixed isolates originated from the Northwest area and were grouped with the clonal lineage (NA-01) in the cluster analysis but were too diverse to be representatives of the clonal lineage (see above).

Virulence analysis. Previous data published on the virulence of $P$. infestans isolates to a differential set of potato cultivars (16) was used to investigate the correlation between genotypic and phenotypic diversity. The phylogenetic tree (Fig. 4) based on SSR markers and sorted by virulence pattern showed the presence of two clusters (I and II). Isolates from cluster I have a maximum of four races against potato differentials compared with isolates from cluster II that have up to seven races. The same race 1.2.3.7 is found in two subregions (Northwest and North areas) and represented high frequency among all races detected (0.097). Among NA-01 isolates, no virulence was found for the resistance genes $5,8,9,10$, and 11. In contrast, isolates found in cluster II showed a much more diverse virulence pattern (Fig. 4).

\section{Discussion}

During the last decade, although late blight epidemics in Tunisia have become more difficult to control, no population structure and diversity analysis was performed. Elsewhere, some studies indicated that, on potato, the $P$. infestans population was changing (9). To genetically characterize the $P$. infestans population, we used the recently published high-resolution set of SSR markers (21) to analyze isolates collected from the most important potato-growing regions in the north of Tunisia.
We found a high level of nuclear diversity within populations of $P$. infestans in Tunisia combined with a low diversity in mtDNA haplotypes. In this study, we found a clear substructure of the $P$. infestans population. We detected clonal lineage NA-01 (A1 mating type, Ia haplotype) present in all sampling regions, found on potato and tomato that showed generally a simple race structure and was sensitive to metalaxyl. This clonal lineage (NA-01) is not similar to any previously published clonal lineage identified in other parts of the world; however, it appears to be present in Egypt as well (D. E. L. Cooke, personal communication). Characteristics of members of this clonal lineage fit the description of $P$. infestans isolates collected in the past by the low virulence complexity and the A1 mating type. Also, the wide geographic distribution and the large number of subclonal variants indicate that these genotypes could be found for a long time in Tunisian fields. Therefore, we hypothesize that this clonal lineage may be old and that we witnessed the displacement of this clonal lineage in Tunisia by a more complex population which includes the A1 and A2 mating type. Such a replacement of a clonal lineage by a more complex population was reported before $(12,13,31,38)$. Interestingly, the potato population in the Northeast $\mathrm{K}$ region seems to be highly genetically diverse. However, similar to the situation of genotype US-1 in Ecuador (28), the NA-01 clonal lineage can still be found on tomato. On another hand, the genetic structure of the population was confirmed by the model-based approach, which showed a clear separation between isolates of the clonal lineage (NA-01) and isolates of the genetic diverse cluster (II). Structure analysis showed high membership of genotypes in their subpopulations for most

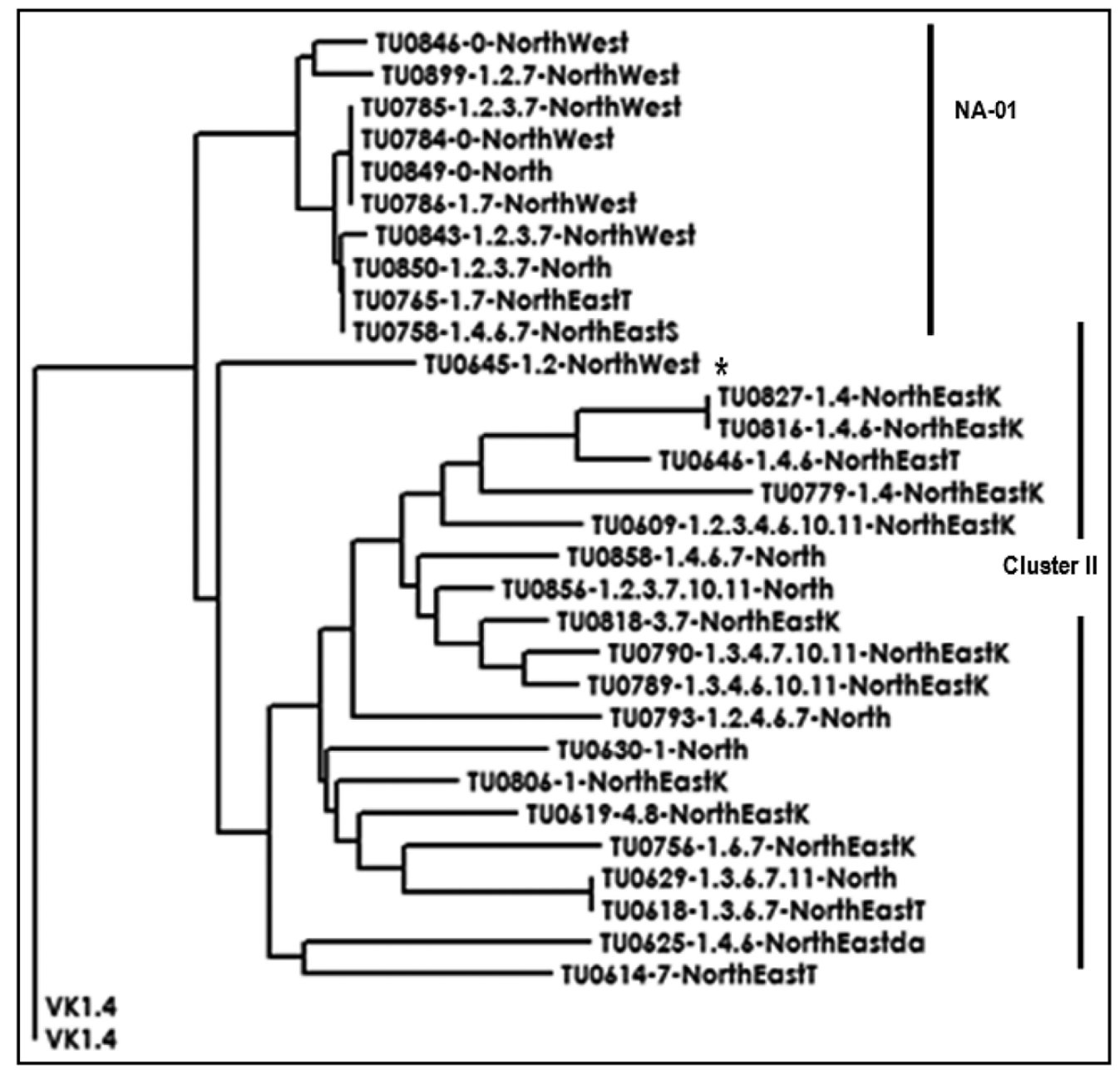

Fig. 4. Phylogenic tree based on simple-sequence repeat patterns sorted by of 30 isolates used in virulence analysis rooted by reference isolate VK 1.4 . Virulence pattern was linked to the isolate's name in the tree (e.g., TU0785-1.2.3.7-Northwest: TU0785 from Northwest that has 1.2.3.7 virulence pattern); * indicates that TU0645 is an admixed isolate belonging to NA-01. 
isolates but three genotypes were characterized as admixed (TU0645, TU0787, and TU0897). Based on this analysis and the detailed analysis of the SSR alleles at each locus (Supplementary Table) we hypothesize that these three isolates may be the result of a sexual cross between a member of the NA-01 clonal lineage and an as-yet-unknown genotype. The identification of these potentially admixed strains contrasts with replacements reported elsewhere, in which no admixed strains derived from a clonal lineage were identified (13).

Isolates of the clonal lineage were detected in all regions, on all hosts, in all seasons, and during all sampling years. This can be the result of asexual propagation during the partly overlapping seasons of potato and the year-round production of tomato. In the genetically highly diverse group, we recovered many genotypes from the same location derived from the same potato genotype. Nevertheless, we found that the same genotypes from the highly diverse group (II) were collected from different seasons. We expect that at least some of these genotypes survived and spread between seasons, demonstrating their potential to start an epidemic. In addition, we noted that the genetic diversity was much higher in the population collected from potato than that from tomato, which may affect the selection. The clear distinction between the tomato and potato isolates corroborate with the study of Legard et al. (20), who reported a host specialization to tomato of isolates from the United States, Canada, Mexico, and the Netherlands.

The isolates from the clonal lineage NA-01 consistently showed less complex races than isolates from the genetically diverse group (15). The presence of both mating types could result in sexual reproduction in Tunisia. Alternatively, these genotypes were derived from a sexual population elsewhere and were migrants, probably coming to Tunisia through imported potato seed, and had a more complex race structure from the start. Elsewhere, high virulence diversity was reported in many countries in the world $(2,14,30,34)$. Nearly all potato seed for the beginning of the season (early-season and main-season crops) are imported. Seed potato is derived from several European countries but especially from the Netherlands (more than 50\%). Latent infections of tubers are considered to be a clear threat for the spread of $P$. infestans genotypes and are likely the founder of the diverse population in Tunisia. However, genotypes that dominate the European population and, particularly, the Dutch population such as 'genotype-13/Blue-13' and Pink-6 $(10,23,24)$ were not recovered in this current survey, suggesting a clear bottleneck for the spread of these genotypes by imported tubers. Possibly, the imported tubers had only low infection levels and, combined with the low percentage of infected tubers that can start an epidemic (33) and the possible low adaptation of these $P$. infestans genotypes, restricted the migration of these European genotypes. Because 'genotype_13' was recently introduced in Algeria (6) and India (4), probably via infected imported potato seed, we recommend continued monitoring for these genotypes in Tunisia. This genotype is highly aggressive and resistant to metalaxyl; therefore, its introduction to Tunisia may have serious consequences for the efficacy of metalaxyl-based control strategies still applied in some regions in Tunisia (15).

Altogether, Tunisian $P$. infestans population seems to have a clear structure showing the presence of a clonal lineage NA-01 in all sampling regions and the presence of a highly genetic diverse group detected in North and Northeast areas. The possible presence of the diverse population could relate to the increased difficulties that potato growers face in the control of late blight, because members of this population are more virulent and less sensitive to metalaxyl, and a possible sexual cycle could result in highly persistent oospores in the soil. The uncovered dynamics of the P. infestans population in Tunisia compel continued monitoring of this pathogen and may require adaptation of the control strategies for late blight in Tunisia.

\section{Acknowledgments}

We thank the Genetic and Plant Breeding Laboratory (INAT, Tunisia), the International foundation for Science (IFS), and Plant Research International,
Wageningen UR (The Netherlands) for the financial support; the Tunisian Potato Centre of Saida (CTPT) for ensuring the sampling; and W. Jmour, I. Fekih, and I. Hannachi in Tunisia and M. van Gent-Pelzer, T. van den Bosch, H. Rietman, and D. Huigen in the Netherlands for technical support.

\section{Literature Cited}

1. Agapow, P.-M., and Burt, A. 2001. Indices of multilocus linkage disequilibrium. Mol. Ecol. Notes 1:101-102.

2. Bouws, H., and Finckh, E. M. 2007. Effects of cropping history and origin of seed potatoes on population structure of Phytophthora infestans. Eur. J. Plant Pathol. 117:313-327.

3. Campana, M. G., Hunt, H. V., Jones, H., and White, J. 2010.CorrSieve: software for summarizing and evaluating Structure output. Mol. Ecol. Resour. 11:349-352.

4. Chowdappa, P., Kumar, N. B. J., Madhura, S., Kumar, M. S. P., Myers, K. L., Fry, W. E., Squires, J. N., and Cooke, D. E. L. 2013. Emergence of 13_A2 Blue lineage of Phytophthora infestans was responsible for severe outbreaks of late blight on tomato in South-West India. J. Phytopathol. 161:49-58.

5. Cooke, L. R., Schepers, H. T. A. M., Hermansen, A., Bain, R. A., Bradshaw, N. J., Ritchie, F., Shaw, D. S., Evenhuis, A., Kessel, G. J. T., Wander, J. G. N., Andersson, B., Hansen, J. G., Hannukkala, A., Nærstad, R., and Nielsen, B. J.2011. Epidemiology and integrated control of potato late blight in Europe. Potato Res. 54:183-222.

6. Corbière, R., Rekad, F. Z., Galfout, A., Andrivon, D., and Bouznard, Z. 2010. Phenotypic and genotypic characteristics of Algerian isolates of Phytophthora infestans. Proc. 12th Euroblight Workshop, Arras, France. PPO Spec. Rep. No. 14:133-146.

7. Excoffier, L., Laval, G., and Schneider, S. 2005. Arlequin ver. 3.0: an integrated software package for population genetics data analysis. Evol. Bioinf. Online 1:47-50.

8. Ferjaoui, S., Romdhani, M. E., Kammassy, N., and Allagui, M. B. 2008 Evaluation de quelques variétés de pomme de terre vis-à-vis du mildiou causé par Phytophthora infestans dans les conditions d'infection naturelle en Tunisie. Ann. INRAT 81:7-23.

9. Fry, W. 2008. Phytophthora infestans: the plant (and $R$ gene) destroyer Mol. Plant Pathol. 9:385-402.

10. Fry, W., Grünwald, N., Cooke. D. E. L., McLeod, A., Forbes. G., and Cao, K. 2009. Population genetics and population diversity of Phytophthora infestans. Pages 139-164 in: Oomycete Genetics and Genomics: Diversity, Interactions, and Research Tools. K. Lamour and S. Kamoun, eds. WileyBlackwell, Hoboken, NJ.

11. Glaubitz, J. C. 2004. Convert: a user-friendly program to reformat diploid genotypic data for commonly used population genetic software packages. Mol. Ecol. Notes 4:309-310.

12. Goodwin, S. B., Cohen, B. A., Deahl, K. L., and Fry, W. E. 1994. Migration from Northern Mexico as the Probable cause of recent genetic changes in populations of Phytophthora infestans in the United States and Canada. Phytopathology 84:553-558.

13. Govers, F., and Gijzen, M. 2006. Phytophthora genomics: the plant destroyers' genome decoded. Mol. Plant-Microbe Interact. 19:1295-1301.

14. Guo, J., van der Lee, T. A. J., Qu, D. Y., Yao, Y. Q., Gong, X. F., Liang, D. L., Xie, K. Y., Wang, X. W., and Govers, F. 2009. Phytophthora infestans isolates from Northern China show high virulence diversity but low genotypic diversity. Plant Biol. 11:57-67.

15. Harbaoui, K., Hamada, W., Vleeshouwers, V. G. A. A., Khammassy, N., Harrabi, M., and van der Lee, T. A. J. 2013. Characterisation of Phytophthora infestans isolates collected from potato and tomato crops in Tunisia during 2006-2008. Pot. Res. Online publication. doi:10.1007/s11540012-9228-3

16. Harbaoui, K., Harrabi, M., Vleeshouwers, V. G. A. A., and Hamada, W. 2011. Virulence patterns of Phytophthora infestans isolates using R differential set of Solanum demissum: a useful tool to identify pathogen races in Tunisia. Tun. J. Plant Prot. 6:1-10.

17. Haverkort, A. J., Boonekamp, P. M., Hutten, R., Jacobsen, E., Lotz, L. A. P., Kessel, G. J. T., Visser, R. G. F., and van der Vossen, E. A. G. 2008.Societal costs of late blight in potato and prospects of durable resistance through Cisgenic modification. Pot. Res. 51:47-57.

18. Jaime-Garcia, R., Orum, T. V., Felix-Gastelum, R., Trinidad-Correa, R., VanEtten, H. D., and Nelson, M. R. 2001. Spatial analysis of Phytophthora infestans genotypes and late blight severity on tomato and potato in the Del Fuerte Valley using geostatistics and geographic information systems. Phytopathology 91:1156-1165.

19. Jmour, W., and Hamada, W. 2006. First report of A2 mating type of Phytophthora infestans in Tunisia using molecular markers and some observations on its metalaxyl resistance. Tun. J. Plant Prot. 1:85-92.

20. Legard, D. E., Lee, T. Y., and Fry, W. E. 1995. Pathogenic specialization in Phytophthora infestans: aggressiveness on tomato. Phytopathology 85:13621367

21. Li, Y., Cooke, D. E. L., Jacobsen, E., and van der Lee, T. A. J. 2013. Efficient multiplex simple sequence repeat genotyping of the oomycete plant pathogen Phytophthora infestans. J. Microbiol. Methods 92:316-322.

22. Li, Y., Govers, F., Mendes, O., Testa, A., Jacobsen, E., Huang, S. W., and 
van der Lee, T. A. J. 2010. A new set of highly informative SSR markers for Phytophthora infestans population analysis assembled into an efficient multiplex. Mol. Ecol. Res. 10:1098-1105.

23. Li, Y., van der Lee, T. A. J., Evenhuis, A., van den Bosch, G. B. M., van Bekkum, P. J., Förch, M. G., van Gent-Pelzer, M. P. E, van Raaij, H. M. G., Jacobsen, E., Huang, S. W., Govers, F., Vleeshouwers, V. G. A. A., and Kessel, G. J. T. 2012. Population dynamics of Phytophthora infestans in the Netherlands reveals expansion and spread of dominant clonal lineages and virulence in sexual offspring. Genes Genomes Genet. 2:1529-1540.

24. Li, Y., van der Lee, T., Zhu, J. H., Jin, G. H., Lan, C. Z., Zhu, S. X., Zhang, R. F., Liu, B. W., Zhao, Z. J., Kessel G. J. T., Huang, S. W., and Jacobsen, E. 2012. Population structure of Phytophthora infestans in China-geographic clusters and presence of the EU genotype Blue_13. Plant Pathol. Online publication. doi:10.1111/j.1365-3059.2012.02687.x

25. McDonald, B. A., and Linde, C. 2002. Pathogen population genetics, evolutionary potential, and durable resistance. Annu. Rev. Phytopathol. 40:349-379.

26. Mukalazi, J., Adipala, E., Sengooba, T., Hakiza, J. J., Olanya, M., and Kidanemariam, H. M. 2001. Metalaxyl resistance, mating type and pathogenicity of Phytophthora infestans in Uganda. Crop Prot. 20:379-388.

27. Nei, M. 1973. The theory and estimation of genetic distance. Pages 45-54 in: Genetic Structure of Populations. N. E. Morton, ed. University of Hawaii Press, Honolulu.

28. Oyarzun, P. J., Pozo, A., Ordoñez, M. E., Doucett, K., and Forbes, G.A. 1998. Host specificity of Phytophthora infestans on tomato and potato in Ecuador. Phytopathology 88:265-71.

29. Pritchard, J. K., Stephens, M., and Donnelly, P. 2000. Inference of population structure using multilocus genotype data. Genetics 155:945-959.
30. Runno-Paurson, E., Fry, W. E., Myers, K. L., Koppel, M., and Mänd, M. 2009. Characterization of Phytophthora infestans isolates collected from potato in Estonia during 2002-2004. Eur. J. Plant Pathol. 124:565-575.

31. Spielman, L. J. 1991. Isoenzymes and population genetics of Phytophthora infestans. Pages 231-241 in: Phytophthora. R. C. Shattock, D. S. Shaw, L. R. Cook, and J. A. Lucas, eds. Cambridge University Press, Cambridge.

32. Van de Peer, Y., and De Wachter, R. 1994. TREECON for Windows: a software package for the construction and drawing of evolutionary trees for the Microsoft Windows environment. Comput. Appl. Biosci. 10:569-570.

33. Van der Zaag, D. E. 1956. Overwintering en epidemiologie van Phy tophthora infestans, tevens enige nieuwe bestrijdingsmogelijkheden Tijdschr. Plantenziekten 62:89-156.

34. Van Poppel, P. M. J. A., Huigen, D. J., and Govers, F. 2009. Differential recognition of Phytophthora infestans races in potato R4 breeding lines. Phytopathology 99:1150-1155.

35. Woodham-Smith, C. 1962. The Great Hunger. Harper and Row, New York.

36. Yeh, F. C., Yang, R. C., Boyle, T. B. J., Ye, Z. H., and Mao, J. X. 1997 POPGENE, the User-friendly Shareware for Population Genetic Analysis. Molecular Biology and Biotechnology Centre, University of Alberta Alberta, Canada.

37. Zhan, J., Pettway, R. E., and McDonald, B. A. 2003. The global genetic structure of the wheat pathogen Mycosphaerella graminicola is characterized by high nuclear diversity, low mitochondrial diversity, regular recombination, and gene flow. Fungal Genet. Biol. 38:286-297.

38. Zwankhuizen, M. J., Govers, F., and Zadoks, J. C. 2000. Inoculum sources and genotypic diversity of Phytophthora infestans in Southern Flevoland, the Netherlands. Eur. J. Plant Pathol. 106:667-80. 